Check for updates

Cite this: RSC Adv., 2018, 8, 4191

Received 18th December 2017 Accepted 15th January 2018

DOI: $10.1039 / c 7 r a 13436 c$

rsc.li/rsc-advances

\section{Electronic structure, polaron formation, and functional properties in transition-metal tungstates}

\author{
Khang Hoang, (D) a Myungkeun $\mathrm{Oh}^{\mathrm{b}}$ and Yongki Choi (D)*ab
}

Transition-metal tungstates $\mathrm{MWO}_{4}(\mathrm{M}=\mathrm{Co}, \mathrm{Ni}, \mathrm{Cu}, \mathrm{Zn})$ have applications in many areas, including supercapacitors. A good understanding of the electronic structure is essential to understanding their functional properties. Here, we report a first-principles study of the materials using hybrid densityfunctional calculations. The electronic structure is analyzed with a focus on the nature of the electronic states near the band edges. We find that hole polarons can form at the Co lattice site in $\mathrm{CoWO}_{4}$ and the $\mathrm{O}$ site in $\mathrm{NiWO}_{4}, \mathrm{CuWO}_{4}$, and $\mathrm{ZnWO}_{4}$, resulting in the formation of $\mathrm{Co}^{3+}$ in the former and $\mathrm{O}^{-}$in the latter. The electrochemical activity observed in certain tungstate compounds, but not in others, appears to be related to the ability to form hole polarons on the transition-metal ions. The formation energy and migration barrier of the hole polaron in $\mathrm{CoWO}_{4}$ are also calculated and the results are employed to understand the reported $p$-type conductivity.

\section{Introduction}

Transition-metal tungstates $\mathrm{MWO}_{4}(\mathrm{M}=\mathrm{Co}, \mathrm{Ni}, \mathrm{Cu}, \mathrm{Zn})$ have been of interest for a wide range of applications, including supercapacitors, ${ }^{\mathbf{1 , 2}}$ photocatalysts, ${ }^{3-5}$ scintillators,${ }^{6}$ and sensors. ${ }^{7}$ The compounds possess the monoclinic $(P 2 / c)$ wolframite-type structure ${ }^{8-10}$ see Fig. 1, except $\mathrm{CuWO}_{4}$ whose symmetry is triclinically distorted with the space group $P \overline{1}$ due to the JahnTeller effect associated with the $\mathrm{Cu}^{2+}$ ion. ${ }^{10} \mathrm{CoWO}_{4}$, for example, were shown to be a promising supercapacitor electrode material, ${ }^{1}$ suggesting that the $\mathrm{Co}^{3+/ 2+}$ redox couple may be active. Bharati et al. ${ }^{\mathbf{1 1}}$ also reported that $\mathrm{CoWO}_{4}$ is a "p-type

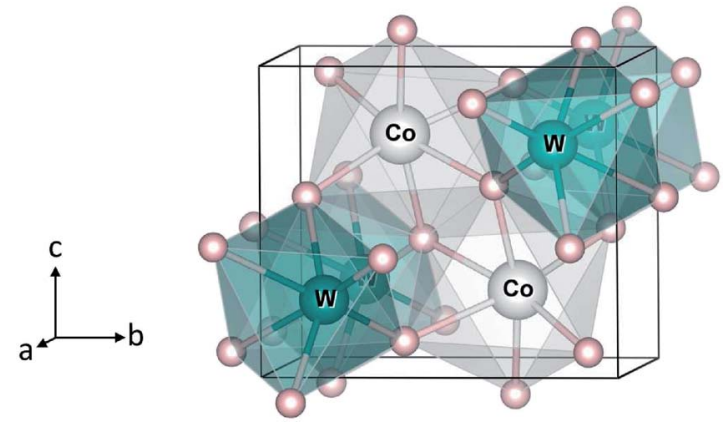

Fig. 1 Crystal structure of monoclinic $\mathrm{CoWO}_{4}$. Large (gray) spheres are Co, medium (blue) spheres are $\mathrm{W}$, and small (red) spheres are $\mathrm{O}$.

a Department of Physics, North Dakota State University, Fargo, North Dakota 58108, USA. E-mail: yongki.choi@ndsu.edu

${ }^{b}$ Materials and Nanotechnology Program, North Dakota State University, Fargo, North Dakota 58105, USA semiconductor" and its electronic conduction is likely to occur through hopping of small polarons. $\mathrm{ZnWO}_{4}$, on the other hand, was found not to show any electroactivity when used as a pseudocapacitive electrode material. ${ }^{12}$ As shown in previous studies, electronic structure and polaron formation can provide crucial information on the electrochemical properties of complex transition-metal oxides. ${ }^{13-15}$

The electronic structure of the tungstates mentioned above has been investigated by several research groups using firstprinciples calculations based on density-functional theory (DFT) within the standard local-density or generalized gradient approximation and/or its extension DFT $+U$ where $U$ is the onsite Hubbard correction. ${ }^{16-19}$ The methods used in these previous studies are, however, often have limited predictive power because the electronic states in the materials (e.g., the transition-metal $\mathrm{d}$ and oxygen $\mathrm{p}$ states) are not treated on equal footing. Besides, to our knowledge, polaron formation in the compounds has not been studied. Recently, two transitionmetal tungstates, $\mathrm{FeWO}_{4}$ and $\mathrm{MnWO}_{4}$, have been studied in detail and the results show that both hole and electron polarons can occur and participate in charge transport and electrochemical processes. ${ }^{15}$ It thus remains to be explored if polarons also form in the other tungstates.

We herein report a first-principles study of $\mathrm{MWO}_{4}(\mathrm{M}=\mathrm{Co}$, $\mathrm{Ni}, \mathrm{Cu}, \mathrm{Zn}$ ) using a hybrid DFT/Hartree-Fock method in which all electronic states in the materials are treated on equal footing. The focus of this work is on the electronic structure, particularly the nature of the electronic states near the band edges, in the different tungstate compounds, and the ability to stabilize an electron and/or hole polaron. We also explicitly investigate the formation of selected intrinsic point defects in $\mathrm{CoWO}_{4}$ as well as the migration of hole polarons and discuss its 
relevance to the observed p-type electronic conduction. The electronic structure of $\mathrm{FeWO}_{4}$ and $\mathrm{MnWO}_{4}$ is also reproduced and included for comparison.

\section{Methods}

The total-energy calculations are based on DFT, using the HeydScuseria-Ernzerhof (HSE06) screened hybrid functional, ${ }^{20}$ the projector augmented wave (PAW) method, ${ }^{21}$ and a plane-wave basis set, as implemented in the Vienna $A b$ Initio Simulation Package (VASP). ${ }^{22}$ The Hartree-Fock mixing parameter and the screening length are set to their standard values of 0.25 and 10 $\AA$, respectively. We use the standard PAW potentials in the VASP database which treat Mn $3 \mathrm{~d}^{6} 4 \mathrm{~s}^{1}$, Fe $3 \mathrm{~d}^{7} 4 \mathrm{~s}^{1}$, Co $3 \mathrm{~d}^{8} 4 \mathrm{~s}^{1}$, Ni $3 \mathrm{~d}^{9}$ $4 \mathrm{~s}^{1}$, Cu $3 \mathrm{~d}^{10} 4 \mathrm{p}^{1}$, Zn $3 \mathrm{~d}^{10} 4 \mathrm{p}^{2}$, W $6 \mathrm{~s}^{2} 5 \mathrm{~d}^{4}$, and O $2 \mathrm{~s}^{2} 2 \mathrm{p}^{4}$ explicitly as valence electrons and the rest as core electrons. The planewave basis-set cutoff is set to $500 \mathrm{eV}$ and spin polarization is included. The calculations of bulk properties in $\mathrm{MWO}_{4}$ (two formula units per unit cell) are carried out using a $5 \times 4 \times 5 \mathrm{k}$ point mesh. Point defects in $\mathrm{CoWO}_{4}$ are modelled using $2 \times 2 \times$ 2 (96-atom) monoclinic supercells. Integrations over the supercell Brillouin zone is carried out using the $T$ point. In all calculations, structural relaxations are performed with the HSE06 functional and the force threshold is chosen to be $0.01 \mathrm{eV} \AA^{-1}$.

The formation energy of a cobalt vacancy $\left(V_{\mathrm{Co}}\right)$ with charge state $q$ in $\mathrm{CoWO}_{4}$ is defined as

$$
E^{\mathrm{f}}\left(V_{\mathrm{Co}}{ }^{q}\right)=E_{\mathrm{tot}}\left(V_{\mathrm{Co}}^{q}\right)-E_{\mathrm{tot}}(\text { bulk })+\mu_{\mathrm{Co}}+q\left(E_{\mathrm{v}}+\mu_{\mathrm{e}}\right)+\Delta^{q},
$$

where $E_{\text {tot }}\left(V_{\mathrm{Co}}{ }^{q}\right)$ and $E_{\text {tot }}($ bulk) are, respectively, the total energy of a supercell containing the vacancy and that of an equivalent supercell of the perfect $\mathrm{CoWO}_{4} \cdot \mu_{\text {Co }}$ is the atomic chemical potential of $\mathrm{Co}$, referenced to the total energy per atom of the Co metal. $\mu_{\mathrm{e}}$ is the electronic chemical potential, i.e., the Fermi level, referenced to the valence-band maximum (VBM) in the bulk $\left(E_{\mathrm{v}}\right) . \Delta^{q}$ is the correction term to align the electrostatic potentials of the bulk and defect supercells and to account for finite-size effects on the total energies of charged defects. ${ }^{23}$ The expression for the formation energy of a small hole polaron $(q=$ +1 ) is similar to eqn (1), except that the atomic chemical potential term is zero since there is only the exchange of an electron with its reservoir. In eqn (1), vibrational contributions to the energies are neglected because they are small or negligible. ${ }^{24}$ Besides, significant cancellation occurs between different terms in the equation.

\section{Results and discussion}

\subsection{Atomic and electronic structure}

We start with the bulk properties of the tungstates. Fig. 1 shows the relaxed structure of monoclinic $\mathrm{CoWO}_{4}$; the structure of $\mathrm{NiWO}_{4}$ and $\mathrm{ZnWO}_{4}$ is similar. The lattice parameters of $\mathrm{MWO}_{4}$ are listed in Table 1. We find that the calculated values are in excellent agreement with the reported experimental ones. ${ }^{8-10}$

In $\mathrm{CoWO}_{4}$, Co is found to be stable as high-spin $\mathrm{Co}^{2+}$ with a magnetic moment of $2.73 \mu_{\mathrm{B}}$; $\mathrm{W}$ is stable as $\mathrm{W}^{6+}$. Our
Table 1 Lattice parameters of tungstates $\mathrm{MWO}_{4}(\mathrm{M}=\mathrm{Co}, \mathrm{Ni}, \mathrm{Cu}, \mathrm{Zn})$

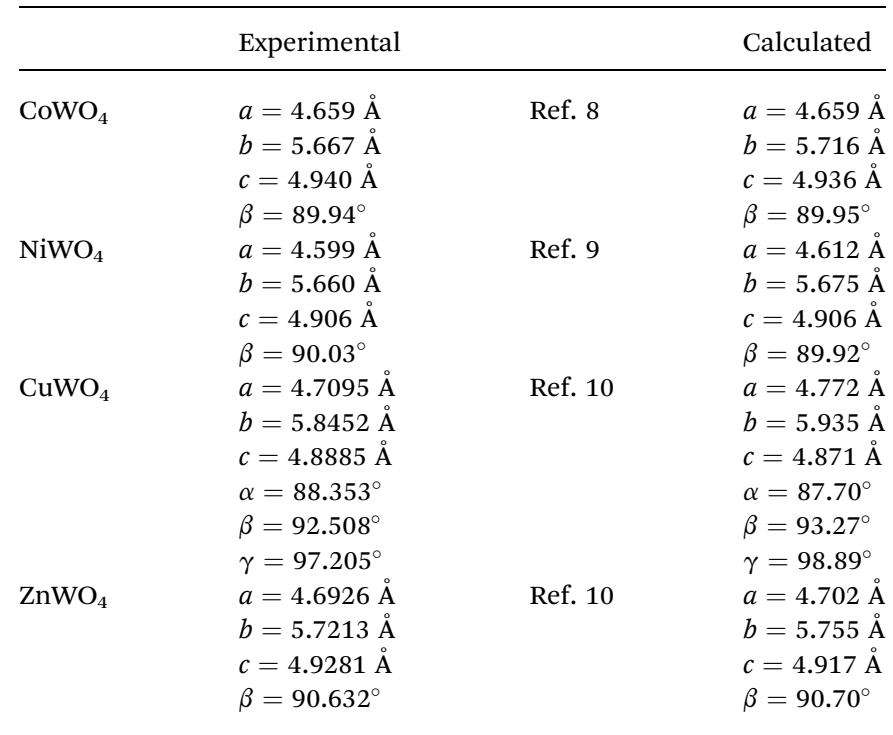

calculations using $2 \times 1 \times 1$ supercells of the unit cell shown in Fig. 1, similar to the $2 \times 1 \times 1$ models for $\mathrm{FeWO}_{4}$ and $\mathrm{MnWO}_{4}$ described in ref. 15, indicate that the ferromagnetic (FM) and antiferromagnetic (AF) spin configurations are degenerate in energy.

Fig. 2(a) shows the total and projected electronic density of states of $\mathrm{CoWO}_{4}$. Focusing on the electronic structure near the band edges, we find that the VBM is predominantly composed of the Co $3 \mathrm{~d}$ states, whereas the conduction-band minimum (CBM) is predominantly the $\mathrm{W} 5 \mathrm{~d}$ and Co $3 \mathrm{~d}$ states. A detailed analysis of the electronic structure shows that each of the two Co atoms in the unit cell accounts for $39 \%$ of the electronic states at the VBM; at the CBM, each $\mathrm{W}$ accounts for $32 \%$ and each Co contributes $15 \%$. The calculated band gap is $3.10 \mathrm{eV}$, a direct gap at the $\Gamma$ point. This value is very close to the experimental value $2.80 \mathrm{eV}$ estimated by Bharati et al., ${ }^{11} \mathrm{ob}-$ tained by assuming that the electronic conduction above $750 \mathrm{~K}$ is through band-like carriers.

As for $\mathrm{NiWO}_{4}$, $\mathrm{Ni}$ is stable as $\mathrm{Ni}^{2+}$ with a calculated magnetic moment of $1.74 \mu_{\mathrm{B}}$. The FM and AF spin configurations $\mathrm{NiWO}_{4}$ are almost degenerate in energy; the FM configuration is higher in energy than the AF configuration with parallel spins within the Ni zigzag chains along the $c$ axis but with adjacent chains coupled antiferromagnetically by only $8 \mathrm{meV}$ per formula unit. Fig. 2(b) shows the electronic structure of $\mathrm{NiWO}_{4}$. The VBM of the compound is predominantly composed of the Ni $3 \mathrm{~d}$ states and the $\mathrm{O} 2 \mathrm{p}$ states; each $\mathrm{Ni}$ atom in the unit cell accounts for $16 \%$ of the electronic states at the VBM and some $\mathrm{O}$ atoms contribute up to $10 \%$ each. The CBM, on the other hand, is predominantly composed of the $\mathrm{W} 5 \mathrm{~d}$ states and the $\mathrm{Ni} 3 \mathrm{~d}$ states; each $\mathrm{W}$ atom accounts for $28 \%$ and each $\mathrm{Ni}$ atom contributes $20 \%$. $\mathrm{NiWO}_{4}$ has an indirect band gap of $3.41 \mathrm{eV}$. Experimentally, the compound was reported to have an optical band gap of $3.2 \pm 0.2 \mathrm{eV} .^{9}$

In $\mathrm{CuWO}_{4}, \mathrm{Cu}$ is found to be stable as $\mathrm{Cu}^{2+}$ with a magnetic moment of $0.76 \mu_{\mathrm{B}}$. The electronic structure of the compound is 


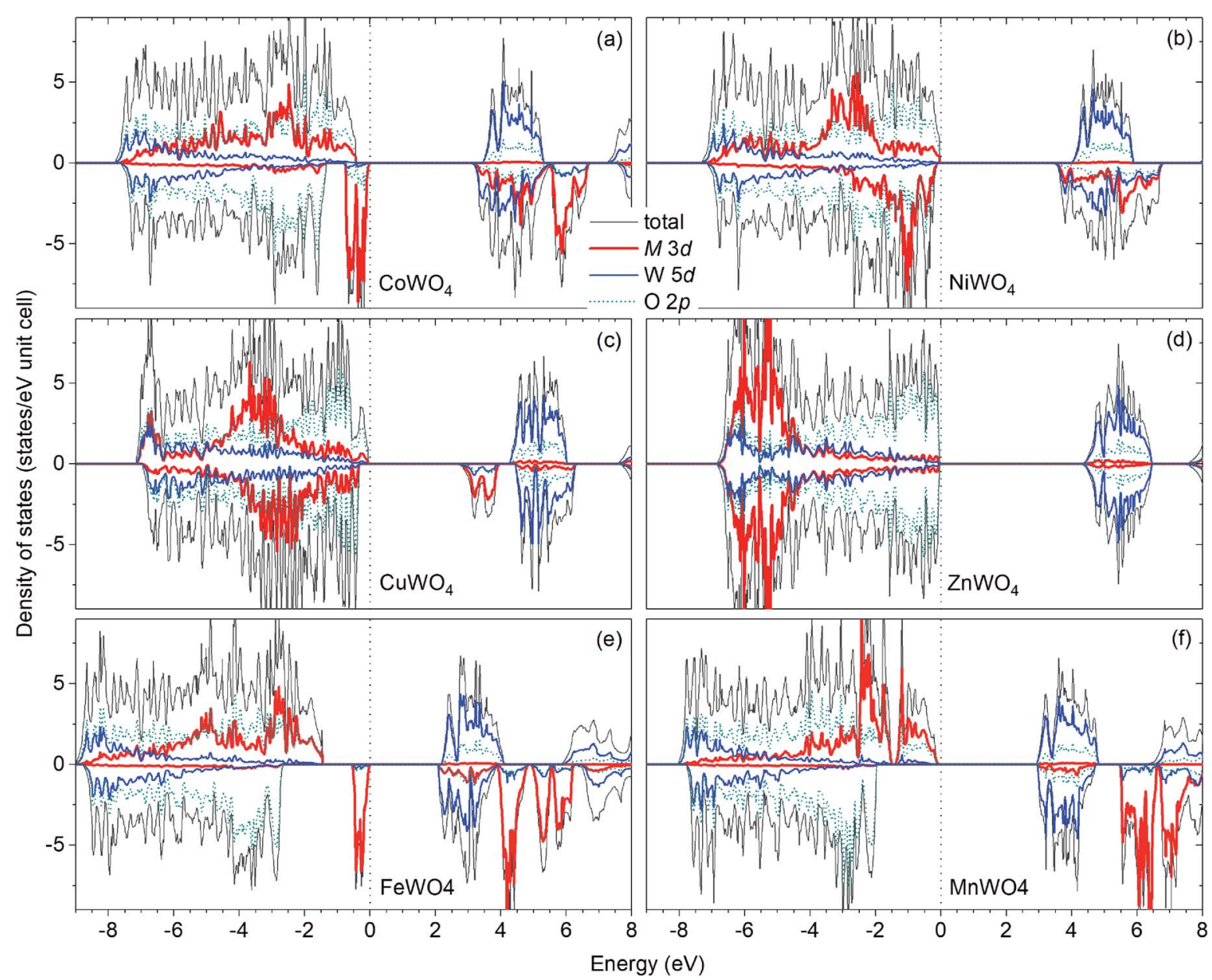

Fig. 2 Total and projected electronic density of states of $\mathrm{MWO}_{4}$ in the ferromagnetic spin configuration: (a) $\mathrm{M}=\mathrm{Co}$, (b) $\mathrm{M}=\mathrm{Ni}$, (c) $\mathrm{M}=\mathrm{Cu}$, and (d) $\mathrm{M}=\mathrm{Zn}$. The results for (e) $\mathrm{FeWO}_{4}$ and (f) $\mathrm{MnWO}_{4}$ are also included for comparison. The zero of energy is set to the highest occupied states.

shown in Fig. 2(c). We find that the VBM is predominantly composed of the $\mathrm{O} 2 \mathrm{p}$ states and some contribution from the $\mathrm{Cu}$ $3 \mathrm{~d}$ states; specifically, the electronic states at the VBM have $12 \%$ from each $\mathrm{Cu}$ atom and some $\mathrm{O}$ atoms contributes up to $13 \%$ each. The CBM is, on the other hand, predominantly the $\mathrm{Cu} 3 \mathrm{~d}$ states ( $29 \%$ from each $\mathrm{Cu}$ atom) and the $\mathrm{W} 5 \mathrm{~d}$ states ( $17 \%$ from each $\mathrm{W}$ atom). $\mathrm{CuWO}_{4}$ is found to have an indirect band gap of $2.71 \mathrm{eV}$, comparable to the reported experimental value $2.3 \mathrm{eV}^{4}$

Finally, $\mathrm{Zn}$ in $\mathrm{ZnWO}_{4}$ is found to be stable as $\mathrm{Zn}^{2+}$ with a zero magnetic moment. Fig. 2(d) shows the electronic structure of $\mathrm{ZnWO}_{4}$. We find that the VBM is predominantly composed of the $\mathrm{O} 2$ ps states; some $\mathrm{O}$ atoms contribute up to $20 \%$ to the electronic states at the VBM. The CBM is, on the other hand, predominantly the $\mathrm{W} 5 \mathrm{~d}$ states (each $\mathrm{W}$ atom contributes $40 \%$ ) and there is a small contribution from the $\mathrm{Zn} 3 \mathrm{~d}$ states (5\% is from each $\mathrm{Zn}$ atom). $\mathrm{ZnWO}_{4}$ has a calculated (direct) band gap of $4.30 \mathrm{eV}$, comparable to the reported experimental value $3.98 \mathrm{eV}^{16}$

For comparison, the results for $\mathrm{FeWO}_{4}$ and $\mathrm{MnWO}_{4}$ are also included; see Fig. 2(e and f). As also reported ref. 15, the VBM of $\mathrm{FeWO}_{4}$ is predominantly composed of the $\mathrm{Fe} 3 \mathrm{~d}$ states with each Fe atom accounts for $46 \%$ of the electronic states at the VBM; the CBM is predominantly the $\mathrm{W} 5 \mathrm{~d}$ states with each $\mathrm{W}$ atom contributes $40 \%$. The VBM of $\mathrm{MnWO}_{4}$ is, on the other hand, predominantly the Mn 3d states with each Mn atom accounts for $27 \%$; the CBM is predominantly composed of the $\mathrm{W} 5 \mathrm{~d}$ states with each $\mathrm{W}$ atom accounts for $39 \%$ of the states at the CBM.

The nature of the electronic structure near the band edges is thus different for different tungstates in the series, as expected. More importantly, our analysis provides a quantitative understanding of electronic structure formation. In the next section, we will examine the implications on defect formation in the materials, particularly on possible formation of polarons.

\subsection{Hole polaron formation}

Calculations for polarons are carried out using the supercell models described in Section 2. For each compound, the creation of a free hole polaron involves removing one electron from the supercell, specifically from the highest occupied state; the creation of a free electron polaron, on the other hand, involves adding one electron to the supercell, i.e., to the lowest unoccupied state. The procedures are performed via controlling the total number of valence electrons in the system. The actual 
occupation of the removed or added electron is checked by examining changes in the charge density, local lattice environment, and magnetic moment. The removal and addition of electrons can be employed to represent oxidation and reduction processes, respectively. ${ }^{\mathbf{1 3 - 1 5}}$

We find that the removal of an electron from the $\mathrm{CoWO}_{4}$ supercell results in a high-spin $\mathrm{Co}^{3+}$ with a calculated magnetic moment of $3.16 \mu_{\mathrm{B}}$, which can be regarded as a highly localized electron hole at one of the Co lattice sites. The local lattice environment is slightly distorted in the presence of the hole; the average $\mathrm{Co}^{3+}-\mathrm{O}$ bond length is $2.02 \AA$, compared to $2.10 \AA$ of the $\mathrm{Co}-\mathrm{O}$ bonds in the bulk. Since the lattice distortion is mainly within the first nearest neighbors of the hole, the localized hole can be regarded as a small hole polaron, hereafter denoted as $\eta_{\text {Co }}{ }^{+}$; see Fig. 3(a). The self-trapping energy $\left(E_{\mathrm{ST}}\right)$, defined as the formation-energy difference between the free hole and the hole polaron, is calculated to be $0.32 \mathrm{eV}$ for $\eta_{\mathrm{Co}}{ }^{+}$. We note that the low-spin $\mathrm{Co}^{3+}\left(0 \mu_{\mathrm{B}}\right)$ solution is higher in energy than the highspin configuration by $0.50 \mathrm{eV}$. The formation of the hole polaron $\eta_{\mathrm{Co}}{ }^{+}$in $\mathrm{CoWO}_{4}$ can be understood in terms of the electronic structure discussed earlier according to which the electron has to be removed from the Co 3d states; see also Fig. 2(a).

The removal of an electron from the $\mathrm{NiWO}_{4}, \mathrm{CuWO}_{4}$, or $\mathrm{ZnWO}_{4}$ supercell, on the other hand, results in a highly localized electron hole at one of the $\mathrm{O}$ sites; i.e., one $\mathrm{O}^{2-}$ is oxidized to $\mathrm{O}^{-}$with a magnetic moment of $\sim 0.7 \mu_{\mathrm{B}}$. The local lattice environment is also distorted in the presence of this hole. $\mathrm{O}^{-}$ can thus also be regarded as a small hole polaron, hereafter denoted as $\eta_{\mathrm{O}}{ }^{+}$. Fig. 3(b) shows the charge density associated with $\eta_{\mathrm{O}}{ }^{+}$in $\mathrm{NiWO}_{4}$. The self-trapping energy is $E_{\mathrm{ST}}=0.22 \mathrm{eV}$ (in $\left.\mathrm{NiWO}_{4}\right), 0.05 \mathrm{eV}\left(\mathrm{CuWO}_{4}\right)$, or $0.38 \mathrm{eV}\left(\mathrm{ZnWO}_{4}\right)$. The very small $E_{\mathrm{ST}}$ value in the case of triclinic $\mathrm{CuWO}_{4}$ could be due to the Jahn-Teller distortion in the material's lattice environment. The main difference between these three tungstates and $\mathrm{CoWO}_{4}$ (as well as $\mathrm{FeWO}_{4}$ and $\left.\mathrm{MnWO}_{4}\right)^{15}$ is thus that the oxidation occurs on the transition metal in the latter whereas it can occur on the oxygen in the former. The results are therefore consistent with the electronic structure presented earlier in which the VBM of $\mathrm{NiWO}_{4}, \mathrm{CuWO}_{4}$, and $\mathrm{ZnWO}_{4}$ is composed mainly of the O $2 \mathrm{p}$
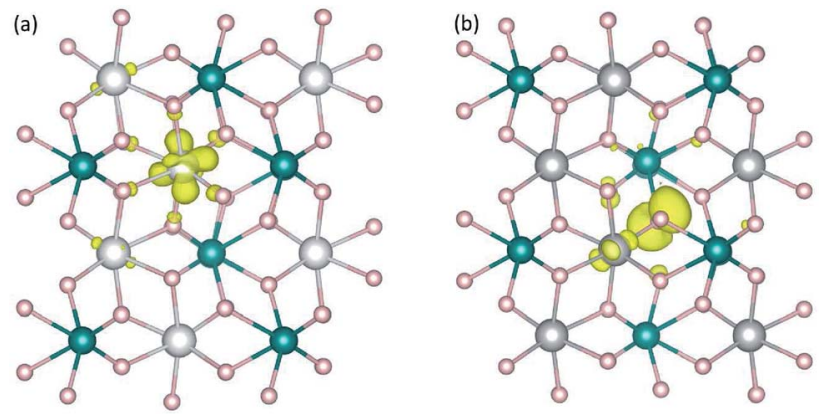

Fig. 3 Charge densities associated with hole polaron (a) $\eta_{\mathrm{Co}^{+}}{ }^{+}$in $\mathrm{CoWO}_{4}$ and (b) $\eta_{\circ}{ }^{+}$in $\mathrm{NiWO}_{4}$. The isovalue for the isosurface (yellow) is set to $0.05 e / \AA^{3}$. Large (gray) spheres are Co or $\mathrm{Ni}$, medium (blue) spheres are $\mathrm{W}$, and small (red) spheres are $\mathrm{O}$. states. We note that, though a free hole polaron associated with $\mathrm{Ni}^{3+}$ cannot be stabilized in bulk $\mathrm{NiWO}_{4}$, it remains to be explored if it can occur in the presence of other defects or at/ near the surface or interface where the lattice environment is different from that in the bulk.

Regarding electron polarons, we find that the addition of an electron to $\mathrm{MWO}_{4}(\mathrm{M}=\mathrm{Co}, \mathrm{Ni}, \mathrm{Cu}, \mathrm{Zn})$ results in an electron that is delocalized all over the supercell. The electron polaron associated with the reduction of $\mathrm{W}^{6+}$ to $\mathrm{W}^{5+}$ is thus not stable in these compounds, unlike in $\mathrm{FeWO}_{4}$ and $\mathrm{MnWO}_{4} \cdot{ }^{15}$ The difference can be traced back to the electronic structure discussed earlier: there is a strong mixing between the $\mathrm{M} 3 \mathrm{~d}$ and $\mathrm{W} 5 \mathrm{~d}$ states at the $\mathrm{CBM}$ of $\mathrm{MWO}_{4}(\mathrm{M}=\mathrm{Co}, \mathrm{Ni}, \mathrm{Cu}, \mathrm{Zn})$. As a result, an electron when added to the materials (e.g., during a reduction process) cannot be localized on any particular $\mathrm{W}$ ion. In the case of $\mathrm{MWO}_{4}(\mathrm{M}=\mathrm{Fe}, \mathrm{Mn})$, the CBM is predominantly composed of the $\mathrm{W} 5 \mathrm{~d}$ states, making it possible for the added electron to be localized..$^{15}$

Finally, as mentioned earlier, some transition-metal tungstates such as $\mathrm{CoWO}_{4}$ (as well as $\mathrm{FeWO}_{4}$ and $\mathrm{MnWO}_{4}$ ) are electroactive, ${ }^{\mathbf{1 , 2 , 2 5}}$ whereas other tungstates such as $\mathrm{ZnWO}_{4}$ are not active, ${ }^{12}$ when used as pseudocapacitive electrode materials. This can be ascribed to the fact that hole polarons associated with the transition metal can form in the former but not in the latter; i.e., the transition-metal (M) redox couple is active in $\mathrm{MWO}_{4}(\mathrm{M}=\mathrm{Co}, \mathrm{Fe}, \mathrm{Mn})$. Given the interplay between the electronic structure and polaron formation, our work thus illustrates how a material's functional properties can be related to its electronic structure.

\subsection{Electronic conduction in $\mathrm{CoWO}_{4}$}

Let us now discuss in more detail the case of $\mathrm{CoWO}_{4}$ where the hole polaron $\eta_{\mathrm{Co}}{ }^{+}$is found to be stable. This defect can occur in the material in combination with other intrinsic point defects. Based on the results previously reported for $\mathrm{FeWO}_{4}$ and $\mathrm{MnWO}_{4},{ }^{15}$ we consider only cobalt vacancies $\left(V_{\mathrm{Co}}\right)$ since $V_{\mathrm{Co}}{ }^{2-}$ (i.e., the removal of an $\mathrm{Co}^{2+}$ ion) is likely the lowest-energy negatively charged intrinsic point defect. Fig. 4 shows the calculated formation energies of $\eta_{\mathrm{Co}}{ }^{+}$and $V_{\mathrm{Co}}$ (in charge states $2-,-$, and 0 ). The slope in the energy plots indicates the charge state: positively (negatively) charged defects have positive (negative) slopes. The formation energies are obtained by assuming that $\mathrm{CoWO}_{4}$ is in equilibrium with $\mathrm{WO}_{3}$ (often used as one of the reactants in the synthesis of $\mathrm{CoWO}_{4}$ ) and air at $900{ }^{\circ} \mathrm{C}$ (the conditions under which the material is prepared). ${ }^{17} \mathrm{We}$ note that the $q=-1$ and 0 charge states of $V_{\mathrm{Co}}$, nominally denoted as $V_{\mathrm{Co}}{ }^{-}$and $V_{\mathrm{Co}}{ }^{0}$, are not really stable; $V_{\mathrm{Co}}{ }^{-}\left(V_{\mathrm{Co}}^{0}\right)$ is, in fact, a defect complex of $V_{\mathrm{Co}}{ }^{2-}$ and one (two) $\eta_{\mathrm{Co}}{ }^{+}$.

Since $\eta_{\mathrm{Co}}{ }^{+}$and $V_{\mathrm{Co}}{ }^{2-}$ are the dominant intrinsic point defects in the material, assuming the absence of any lower-energy negatively charged intrinsic defects or impurities, they determine the position of the Fermi level, which is at $\mu_{\mathrm{e}}^{\text {int }}=1.67 \mathrm{eV}$, where charge neutrality is maintained; see Fig. 4. At this Fermilevel position, the formation energy of the two defects is $1.41 \mathrm{eV}$. We also estimate the migration barrier $\left(E_{\mathrm{m}}\right)$ of the hole polaron, using the method as described in ref. 15 and references therein, 


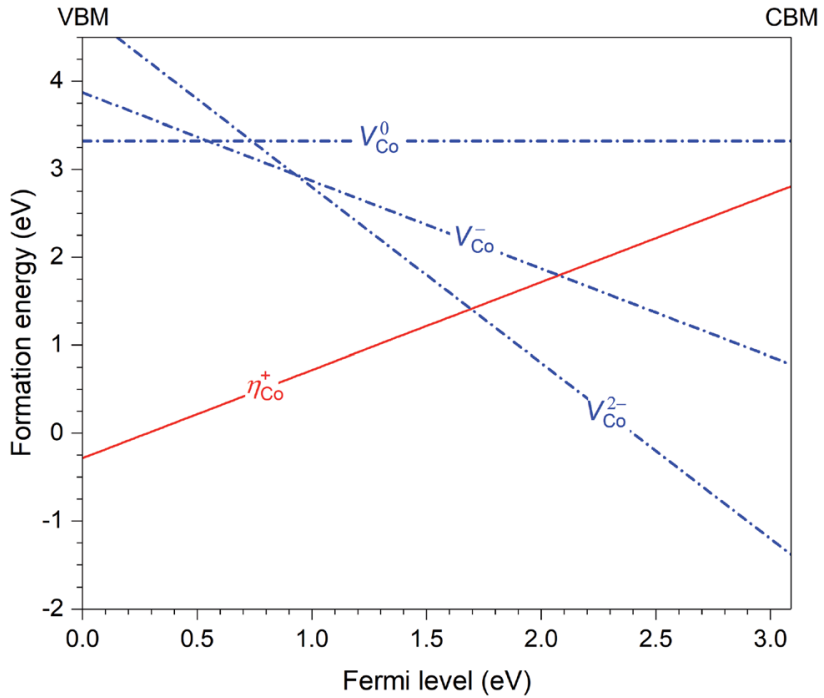

Fig. 4 Formation energies of the hole polaron $\left(\eta_{\mathrm{Co}^{+}}{ }^{+}\right)$and Co vacancies $\left(V_{\mathrm{Co}}\right)$ in $\mathrm{CoWO}_{4}$, plotted as a function of Fermi level from the valence-band maximum (VBM) to the conduction-band minimum (CBM).

and find that $E_{\mathrm{m}}=0.29 \mathrm{eV}$ along the zigzag metal chain (i.e., $c$ axis). For comparison, the migration barrier was reported to be $0.14 \mathrm{eV}$ for $\eta_{\mathrm{Fe}}{ }^{+}$in $\mathrm{FeWO}_{4}$ or $0.28 \mathrm{eV}$ for $\eta_{\mathrm{Mn}}{ }^{+}$in $\mathrm{MnWO}_{4} \cdot{ }^{15}$

Given the low migration barrier, $\eta_{\mathrm{Co}}{ }^{+}$can participate in electronic transport that results in the p-type conductivity as observed in experiments. ${ }^{\mathbf{1 1}}$ Bharati et al. reported that the activation energy in the extrinsic region $(<750 \mathrm{~K})$ is $E_{\mathrm{a}}=0.64 \mathrm{eV}$, whereas $E_{\mathrm{a}}=1.40 \mathrm{eV}$ in the intrinsic region (>750 K). As discussed in detail in ref. 15 and 26, the lower limit of the activation energy is $E_{\mathrm{m}}$ and the upper limit is $E^{\mathrm{f}}+E_{\mathrm{m}}$. The calculated $E_{\mathrm{m}}$ is thus lower than the experimental $E_{\mathrm{a}}$ in the extrinsic region, as expected. In the intrinsic region, our calculations give $E_{\mathrm{a}}=1.70 \mathrm{eV}$. Experimentally, Bharati et al. argued that the conduction is band-type with an activation energy of $1.40 \mathrm{eV} ;^{\mathbf{1 1}}$ however, given that our estimated activation energy is comparable, the conduction may actually still involve hopping of the small hole polarons $\eta_{\mathrm{Co}}{ }^{+}$.

\section{Summary}

A hybrid density-functional study of the electronic structure and polaron formation has been carried out for transition-metal tungstates. The calculated lattice parameters and band gaps are in good agreement with experiments. The nature of the electronic structure at the valence-band top in $\mathrm{CoWO}_{4}$ allows for the formation of hole polarons $\left(\mathrm{Co}^{3+}\right)$ at the transition metal site and hence active $\left(\mathrm{Co}^{3+/ 2+}\right)$ redox couples, similar to what was reported for $\mathrm{FeWO}_{4}$ and $\mathrm{MnWO}_{4}$, whereas in $\mathrm{NiWO}_{4}, \mathrm{CuWO}_{4}$, and $\mathrm{ZnWO}_{4}$ hole polarons $\left(\mathrm{O}^{-}\right)$can be stabilized at the oxygen site. Electron polarons at the $\mathrm{W}$ site cannot be stabilized in $\mathrm{MWO}_{4}(\mathrm{M}=\mathrm{Co}, \mathrm{Ni}, \mathrm{Cu}, \mathrm{Zn})$, unlike in $\mathrm{FeWO}_{4}$ and $\mathrm{MnWO}_{4}$ where the electron polarons were reported to be stable. The difference between the tungstate compounds can be understood in terms of the calculated electronic structure. Finally, we find that hole polarons are responsible for the observed p-type conductivity in $\mathrm{CoWO}_{4}$.

\section{Conflicts of interest}

There are no conflicts to declare.

\section{Acknowledgements}

This work was supported financially by the National Institute of General Medical Sciences of the National Institute of Health under Award No. R15GM122063. The calculations were carried out using computing resources at the Center for Computationally Assisted Science and Technology at North Dakota State University.

\section{References}

1 X. Xing, Y. Gui, G. Zhang and C. Song, Electrochim. Acta, 2015, 157, 15-22.

2 X. Xu, L. Pei, Y. Yang, J. Shen and M. Ye, J. Alloys Compd., 2016, 654, 23-31.

3 H. Jia, J. Stark, L. Q. Zhou, C. Ling, T. Sekito and Z. Markin, RSC Adv., 2012, 2, 10874-10881.

4 C. R. Lhermitte and B. M. Bartlett, Acc. Chem. Res., 2016, 49, 1121-1129.

5 C. Zhang, H. Zhang, K. Zhang, X. Li, Q. Leng and C. Hu, ACS Appl. Mater. Interfaces, 2014, 6, 14423-14432.

6 V. B. Mikhailik, H. Kraus, G. Miller, M. S. Mykhaylyk and D. Wahl, J. Appl. Phys., 2005, 97, 083523.

7 C. M. Gonzalez, X. Du, J. L. Dunford and M. L. Post, Sens. Actuators, B, 2012, 173, 169-176.

8 J. B. Forsyth and C. Wilkinson, J. Phys.: Condens. Matter, 1994, 6, 3073.

9 P. Parhi, T. N. Karthik and V. Manivannan, J. Alloys Compd., 2008, 465, 380-386.

10 P. F. Schofield, K. S. Knight, S. A. T. Redfern and G. Cressey, Acta Crystallogr., Sect. B: Struct. Sci., 1997, 53, 102-112.

11 R. Bharati, R. A. Singh and B. M. Wanklyn, J. Mater. Sci., 1981, 16, 775-779.

12 N. Goubard-Bretesché, O. Crosnier, C. Payen, F. Favier and T. Brousse, Electrochem. Commun., 2015, 57, 61-64.

13 K. Hoang and M. Johannes, Chem. Mater., 2011, 23, 30033013.

14 M. D. Johannes, K. Hoang, J. L. Allen and K. Gaskell, Phys. Rev. B, 2012, 85, 115106.

15 K. Hoang, Phys. Rev. Materials, 2017, 1, 024603.

16 J. Ruiz-Fuertes, S. López-Moreno, J. López-Solano, D. Errandonea, A. Segura, R. Lacomba-Perales, A. Muñoz, S. Radescu, P. Rodríguez-Hernández, M. Gospodinov, L. L. Nagornaya and C. Y. Tu, Phys. Rev. B, 2012, 86, 125202.

17 S. Dey, R. A. Ricciardo, H. L. Cuthbert and P. M. Woodward, Inorg. Chem., 2014, 53, 4394-4399.

18 S. Rajagopal, V. L. Bekenev, D. Nataraj, D. Mangalaraj and O. Y. Khyzhun, J. Alloys Compd., 2010, 496, 61-68.

19 C. Ling, L. Q. Zhou and H. Jia, RSC Adv., 2014, 4, 2469224697. 
20 J. Heyd, G. E. Scuseria and M. Ernzerhof, J. Chem. Phys., 2003, 118, 8207-8215.

21 P. E. Blöchl, Phys. Rev. B, 1994, 50, 17953-17979.

22 G. Kresse and J. Furthmüller, Phys. Rev. B, 1996, 54, 1116911186.

23 C. Freysoldt, J. Neugebauer and C. G. Van de Walle, Phys. Rev. Lett., 2009, 102, 016402.
24 C. Freysoldt, B. Grabowski, T. Hickel, J. Neugebauer, G. Kresse, A. Janotti and C. G. Van de Walle, Rev. Mod. Phys., 2014, 86, 253-305.

25 F. Li, X. Xu, J. Huo and W. Wang, Mater. Chem. Phys., 2015, 167, 22-27.

26 K. Hoang and M. D. Johannes, J. Mater. Chem. A, 2014, 2, 5224-5235. 